\title{
ITERATED NONTANGENTIAL LIMITS
}

BY

\author{
K. GOWRISANKARAN
}

ABSTRACT. For functions $f$ in the Nevanlinna class of $U^{\boldsymbol{n}}$ it is proved that the iterated nontangential limits are equal up to a set of measure zero.

Let $f$ be a holomorphic function on $U^{n}$ in the Nevanlinna class of the polydisc. It is a well-known result that the iterated nontangential limits of $f$ exist at Lebesgue almost every point of the distinguished boundary $T^{n}$. It has been an old problem to decide whether these different iterated limits are the same for different orders of iteration [2]. In 1972, we showed that the moduli of the various iterated limits are the same almost everywhere [1]. And recently as a particular case of a general study of functions defined on partial domains we proved that indeed the problem of Zygmund has an affirmative solution. However, it was pointed out to us by Professors C. S. Herz and J. L. Doob that the problem had already been essentially solved in our earlier paper. The following is the statement and the proof.

THEOREM. Let $f$ be a holomorphic function in the Nevanlinna class of the polydisc. The iterated nontangential limits of $f$ exist and are equal except on a set of Lebesgue measure zero on the distinguished boundary, independent of the order of iteration.

Proof. The existence of the iterated limits is a well-known result and the equality of the moduli, almost everywhere is established in [1].

Now, let $A$ be a countable dense subset of the complex plane $C$. Clearly, $f+a$ belongs to the Nevanlinna class for every $a$ in $C$. Hence the moduli of the iterated nontangential limits of $f+a$ are equal almost everywhere independent of the order of iteration. Hence, if $f_{1}$ and $f_{2}$ denote the iterated nontangential limits for two different orders of iteration, then, except for a set of Lebesgue measure zero on $T^{n},\left|f_{1}+a\right|=\left|f_{2}+a\right|$ for all $a$ in $A$. Since $A$ is dense in $C$ we conclude that $f_{1}=f_{2}$ almost everywhere on $T^{n}$. The proof is complete.

Received by the editors October 3, 1974.

AMS (MOS) subject classifications (1970). Primary 32A07; Secondary 31C05. 


\section{BIBLIOGRAPHY}

1. K. Gowrisankaran, Iterated fine limits and iterated nontangential limits, Trans. Amer. Math. Soc. 173 (1972), 71-92. MR 47 \#489.

2. A. Zygmund, Trigonometrical series. Vol. 2, 2nd rev. ed., Cambridge Univ. Press, Now York, 1959. MR 21 \#6495.

DEPARTMENT OF MATHEMATICS, MCGILL UNIVERSITY, MONTREAL, QUEBEC, CANADA 